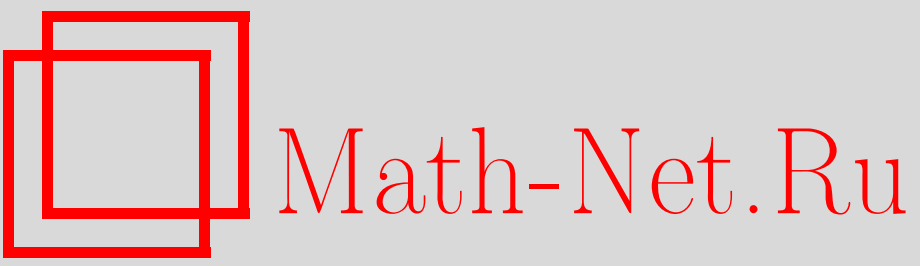

А. А. Смирнов, В. В. Бондарь, О. Д. Роженко, М. В. Мирзоян, А. Д. Держания, Числа Мерсенна в основаниях системы остаточных классов при передаче данных в последовательных каналах связи, Итоги науки и техн. Сер. Соврем. мат. и ее прил. Темат. обз., 2019, том 166, 87-94

DOI: https://doi.org/10.36535/0233-6723-2019-166-87-94

Использование Общероссийского математического портала Math-Net.Ru подразумевает, что вы прочитали и согласны с пользовательским соглашением

http://www.mathnet.ru/rus/agreement

Параметры загрузки:

IP: 18.234 .197 .8

26 апреля 2023 г., 15:56:52 


\title{
ЧИСЛА МЕРСЕННА В ОСНОВАНИЯХ СИСТЕМЫ ОСТАТОЧНЫХ КЛАССОВ ПРИ ПЕРЕДАЧЕ ДАННЫХ В ПОСЛЕДОВАТЕЛЬНЫХ КАНАЛАХ СВЯЗИ
}

(c) 2019 г. А. А. СМИРНОВ, В. В. БОНДАРЬ, О. Д. РОЖЕНКО, М. В. МИРЗОЯН, А. Д. ДАРЖАНИЯ

\begin{abstract}
АннотАция. Рассматривается система остаточных классов для представления данных в каналах связи. В этом случае возможно осуществление обработки данных параллельно без учетов переносов в разрядах. Вместе с тем большая часть каналов связи является последовательной. В связи с этим рассматривается возможность последовательной передачи параллельных данных. Показано, что применение в качестве оснований системы остаточных классов чисел Мерсенна не приводит к снижению скорости передачи данных. Проведена оценка количества информации, содержащейся в позициях чисел позиционного кода.
\end{abstract}

Ключевые слова: число Мерсенна, число Ферма, система остаточных классов, параллельные каналы связи, количество информации.

\section{MERSENNE NUMBERS IN THE BASES OF SYSTEMS OF RESIDUAL CLASSES WHEN TRANSMITTING DATA IN SERIAL COMMUNICATION CHANNELS}

(C) 2019 A. A. SMIRNOV, V. V. BONDAR, O. D. ROZHENKO, M. V. MIRZOYAN, A. D. DARJANIA

\begin{abstract}
In this paper, we consider the system of residual classes for data representation in communication channels. In this case, it is possible to perform parallel data processing without taking into account transfers in bits. However, most of the communication channels are serial; in this regard, the possibility of serial transmission of parallel data is considered. We show that the use of the residual classes of Mersenne numbers as bases of the system does not lead to a decrease in the data transfer rate. The amount of information contained in the positions of the numbers of the positional code is estimated.
\end{abstract}

Keywords and phrases: Mersenne number, Fermat number, system of residual classes, parallel communication channels, amount of information.

AMS Subject Classification: 11A07, 94A17

1. Постановка задачи. В настоящее время все большее распространение при представлении и в обработке данных используются параллельные технологии (см. [1]), например, применение параллельной математики, такой как система остаточных классов (COK) (см. [5,9]). Данные в COK в отличие от позиционной системы счисления (ПСС) представляются в виде параллельных вычетов $\alpha_{i}$ или остатков по выбранным взаимно простым основаниям $p_{i}$ (см. [2]), а операции сложения, вычитания и умножения могут выполняться параллельно по всем вычетам за один 
такт (см. [8]). Например, представим числа $A_{1}=26$ и $A_{2}=17$ в СОК по основаниям $p_{i}=5$, 7,13 . Остатки от деления составят $\alpha_{i}^{\prime}=1,5,0$ и $\alpha_{i}^{\prime \prime}=2,3,4$ соответственно. Сумма чисел в ПСС составит $A_{1}+A_{2}=26+17=43$. Суммирование при этом осуществляется от разряда к разряду, так как $6+7=13$, т.е., есть переносы в старшие разряды. Та же операция суммирования в СОK осуществляется одновременно по всем основаниям согласно выражению

$$
\alpha_{i}^{\prime \prime \prime}=\left(\alpha_{i}^{\prime}+\alpha_{i}^{\prime \prime}\right) \bmod p_{i}
$$

Так, для этих чисел

$$
(1+2) \bmod 5=3 \bmod 5=3, \quad(5+3) \bmod 7=8 \bmod 7=1, \quad(0+4) \bmod 11=4 \bmod 11=4 .
$$

Действительно число 43 в СОК будет $\alpha_{i}^{\prime \prime \prime}=3,1,4$. Еще более эффективной операцией в СОК по сравнению с операцией в ПСС является операция умножения. Так $A_{1} \cdot A_{2}=26 \cdot 17=442$. Очевидно, что на данную операцию в ПСС потребуется еще больше времени, в то время как операция произведения в СОK так же осуществляется одновременно по всем основаниям согласно выражению

Так, для данных чисел

$$
\alpha_{i}^{\prime \prime \prime}=\left(\alpha_{i}^{\prime} \cdot \alpha_{i}^{\prime \prime}\right) \bmod p_{i}
$$

$$
(1 \cdot 2) \bmod 5=2 \bmod 5=2, \quad(5 \cdot 3) \bmod 7=15 \bmod 7=1, \quad(0 \cdot 4) \bmod 13=0 \bmod 13=0 .
$$

Действительно, 442 в СОК по этим основаниям $\alpha_{i}^{\prime \prime \prime}=2,1,0$. Таким образом, преимущество СОК заключается в возможности выполнения операций сложения и умножения параллельно, т. е. без переносов в разрядах числа. При этом числа представляются параллельным набором остатков от деления по $L$ взаимно простым основаниям $p_{i}$, где $i=1, \ldots, L$. Затраты при переводе чисел обратно в ПСС ничтожно малы по сравнению с объемом операций, выполняемых на промежуточных операциях в СОK (см. [6]).

Вместе с тем, преимущественная передача данных как в каналах связи (KC), так и в вычислительных системах осуществляется по последовательным линиям (см. $[4,5])$. Таким образом, к существующим линиям коммуникаций при их проектировании не предъявлялись требования по адаптации к параллельным данным с учетом статистики вычетов (см. [7]). При этом возникает вопрос о скорости передачи параллельных данных по последовательным линиям. Целью данной статьи является оценка возможного снижения скорости передачи данных в COK относительно передачи тех же данных в ПСС по двоичным цифровым линиям.

2. Решение задачи. Данные в СОК представляются по параллельному набору взаимно простых оснований $p_{i}$ в виде вычетов с максимальным значением $a_{i}=p_{i}-1$. Поэтому для передачи таких данных желательно использовать параллельные $\mathrm{KC}$, где по отдельной линии передается значение каждого вычета (остатка). Подобная организация $\mathrm{KC}$ позволит избежать необходимости построения сложных запоминающих устройств и синхронизаторов с аппаратурой, работающей в параллельной математике. Вместе с тем, большинство КС имеет последовательную архитектуру. Передача остатков числа по таким каналам осуществляться последовательно. Очевидно, что выигрышем в данной ситуации является то, что отпадает необходимость использования аппаратуры преобразования данных из канальных данных ПСС в данные, представленные в формате COK для оконечной аппаратуры. Кроме того, COK обладает естественной избыточностью, что обусловлено перекрытием необходимого числового диапазона, обычно кратного $2^{m}$, допустимым числовым диапазоном

$$
P=\prod_{i=1}^{L} p_{i}-1,
$$

не кратным $2^{m}$ (см. [1]), где $m \in \mathbb{N}$ - натуральное число, $L$ - число используемых оснований. Поскольку значение каждого остатка данных COK в последовательном двоичном КС должно передаваться последовательно и в двоичном коде (см. [4]), то возникает вопрос: снизится ли скорость передачи информации в последовательном двоичном КС при передаче по нему данных в формате COK, и если снизится, то на сколько. 
Пусть по $\mathrm{KC}$ необходимо передавать числа диапазона $P(1)$. Тогда в двоичном $\mathrm{KC}$ на передачу каждого числа необходимо использовать $k=\left\lceil\log _{2}(P+1)\right\rceil$ двоичных позиций с учетом передачи нуля, где скобки означают округление до большего целого числа. Например, для передачи семи чисел вычетов от 0 до 6 по основанию 7 необходимо $k=\left\lceil\log _{2}(6+1)\right\rceil=\left\lceil\log _{2} 7\right\rceil=3$ элементарных посылки. При этом максимальный диапазон цифр, включая нуль, передаваемых кодом длиной $k=3$, составляет $P=2^{k}-1=2^{3}-1=7$, т.е. от 0 до 7. Другими словами, образуется избыточность, позволяющая обнаруживать ошибки. Однако максимальное кодовое расстояние, обусловленное операцией округления, меньше двух, поэтому такая избыточность не позволяет обнаруживать все однократные ошибки, она не является эффективной и ведет к потере скорости передачи информации. Поэтому в двоичном КС стараются использовать весь диапазон чисел равный $P=2^{m}-1$. Пусть по двоичному $\mathrm{KC}$ необходимо передавать данные в формате COK с основаниями $p_{1}, p_{2}, \ldots, p_{i}$, где $i=1, \ldots, L$. Таким образом, необходимо передавать систему наименьших неотрицательных вычетов $\alpha_{1}, \alpha_{2}, \ldots, \alpha_{i}$ или остатков по указанным основаниям. Очевидно, что длина кодовой комбинации, необходимая для передачи всех остатков, будет складываться из длин кодовых комбинаций, представленных в двоичной системе счисления, этих остатков. Например, если $p_{i}=2,3,5,7$, то максимальные вычеты по этим основаниям будут иметь значения $\alpha_{i}=p_{i}-1=1,2,4,6$. Для их передачи необходимо передать последовательность из суммы $n_{i}=\left\lceil\log _{2}\left(\alpha_{i}+1\right)\right\rceil$ двоичных кодов или

$$
n(L)=\sum_{i=1}^{L} n_{i}=\sum_{i=1}^{L}\left\lceil\log _{2}\left(\alpha_{i}+1\right)\right\rceil
$$

откуда

$$
n(L)=\sum_{i=1}^{L}\left\lceil\log _{2} p_{i}\right\rceil
$$

Диапазон представления чисел в данной системе оснований определяется (1), поэтому для передачи этого же числового диапазона в ПСС необходимо

$$
k(L)=\left\lceil\log _{2}\left(\prod_{i=1}^{L} p_{i}+1-1\right)\right\rceil=\left\lceil\log _{2}\left(\prod_{i=1}^{L} p_{i}\right)\right\rceil=\left\lceil\sum_{i=1}^{L} \log _{2} p_{i}\right\rceil
$$

двоичных символов. Очевидно, что $n(L)$ и $k(L)$ не равны между собой. Поэтому скорости передачи информации с использованием этих кодов возможно также будут различны. Определим разность выражений (2) и (3), которая определяет потерю производительности источника сообщения (ИС) информации в формате СОK по двоичным $\mathrm{KC}$ :

$$
\Theta(L)=n(L)-k(L) .
$$

Операция округления в выражении (2) означает дополнение числа $p_{i}$ до числа, кратного $2^{m}$. Округление, в физическом смысле, приводит к неоправданной избыточности кода и снижению скорости передачи информации, т.е. появляются неразрешенные кодограммы. Этот очевидный факт можно сформулировать в виде теоремы.

Теорема 1. Максимальный вычет любого простого числа кодируется в двоичном коде с избыточностьюо как минимум в одну кодограмму.

Доказательство. Поскольку все, за исключением 2, взаимно простые основания не кратны числам $2^{m}$, которые кодируются без избытка, то округление всегда будет иметь место. Отметим, что наиболее близкими взаимно простыми числами к числам $2^{m}$ могут быть числа Мерсенна $2^{m}-1$ или числа Ферма $2^{2^{m}}+1$ (см. [9]), где $m$ - натуральное число. Однако первые могут быть закодированы с минимальным избытком, а вторые с максимальным избытком, так как округление (2) осуществляется в большую сторону, кратную $2^{m}$. Таким образом, весь диапазон остатков может быть закодированы только с избыточностью. Если в качестве оснований использовать взаимно простые числа Мерсенна $p_{i}=2^{m}-1$ для $m \geqslant 2$, то указанное дополнение будет минимальным, т.е. 1. Доказательство закончено. 
Далее рассмотрим все числа $p_{i}=2^{m}-1$ для любых $m \geqslant 2$, включающие в себя и числа Мерсенна. В этом случае числовой диапазон СОK составит

$$
P=\prod_{m=2}^{L+1}\left(2^{m}-1\right)-1 .
$$

Здесь верхний предел сумм $L+1$ обусловлен тем, что $m \geqslant 2$. С учетом

$$
\left\lceil\log _{2} p\right\rceil=\left\lceil\log _{2}\left(2^{m}-1\right)\right\rceil=\log _{2} 2^{m}=m,
$$

минимально необходимое число бит (2) для представления чисел СОК в двоичной ПСС составит

$$
n(L)=\sum_{i=1}^{L}\left\lceil\log _{2} p_{i}\right\rceil=\sum_{m=2}^{L+1}\left\lceil\log _{2}\left(2^{m}-1\right)\right\rceil=\sum_{m=2}^{L+1} \log _{2} 2^{m}=\log _{2} \prod_{m=2}^{L+1} 2^{m}=\sum_{m=2}^{L+1} m .
$$

Необходимая длина двоичного кода для передачи того же числового диапазона в формате COK по двоичным КС согласно (3) определяется как

$$
k(L)=\left\lceil\log _{2} \prod_{m=2}^{L+1}\left(2^{m}-1\right)\right\rceil=\left\lceil\sum_{m=2}^{L+1} \log _{2}\left(2^{m}-1\right)\right\rceil .
$$

Поскольку числа $p_{i}=2^{m}-1$ не кратны $2^{m}$, следовательно, их произведение

$$
\prod_{m=1}^{L+1}\left(2^{m}-1\right)
$$

не может быть представлено в виде $2^{l}$, где $l \in \mathbb{N}$. Поэтому округление также будет иметь место. С учетом этого и (6) выражение (4) можно записать как

$$
\Theta(L)=\sum_{m=2}^{L+1} \log _{2} 2^{m}-\left\lceil\sum_{m=2}^{L+1} \log _{2}\left(2^{m}-1\right)\right\rceil .
$$

Очевидно, величина округления в выражении (7) зависит от величины

$$
\Omega(L)=\sum_{m=2}^{L+1} \log _{2} 2^{m}-\sum_{m=2}^{L+1} \log _{2}\left(2^{m}-1\right)
$$

Оценим данную разницу. Оба ряда в выражение (9) знакоположительные, поэтому

$$
\sum_{m=2}^{L+1} \log _{2} 2^{m}-\sum_{m=2}^{L+1} \log _{2}\left(2^{m}-1\right)=\sum_{m=2}^{L+1}\left(\log _{2} 2^{m}-\log _{2}\left(2^{m}-1\right)\right)=\sum_{m=2}^{L+1} \log _{2} \frac{2^{m}}{2^{m}-1} .
$$

Оценим сумму данного ряда. Этот ряд знакоположительный с возрастающей суммой

$$
\Omega(L)>\Omega(1)=\log _{2} \frac{2^{2}}{2^{2}-1} \approx 0,4 .
$$

Заметим, что сумма известного ряда

$$
\sum_{m=2}^{\infty} \frac{1}{2^{m-1}}=1
$$

Сравним почленно эти ряды. Покажем, что

$$
\log _{2} \frac{2^{m}}{2^{m}-1}<\frac{1}{2^{m-1}} .
$$

Последнее запишем в виде

$$
\frac{2^{m}}{2^{m}-1}<2^{2 / 2^{m}} \quad \text { или } \quad 1<2^{2 / 2^{m}}-2^{2 / 2^{m}-m} .
$$


Данное неравенство справедливо для всех $m \geqslant 2$. Так, при $m=2$ получим $1<\sqrt{2}-\sqrt{2} / 4 \approx 1,06$, а при $m \rightarrow \infty-$

$$
\lim _{m \rightarrow \infty} 2^{2 / 2^{m}}\left(1-2^{-m}\right)=1 .
$$

Сделаем вывод, что сумма ряда $(9)$ составляет $0,4<\Omega(L)<1$. Но тогда первая сумма разности (9) превышает вторую сумму этой же разности не более чем на 1 . Поскольку первая сумма в (8) - целое число, в результате округления второй суммы (8) получим $\Theta(L)=0$. Приведенные рассуждения проведены для множества всех чисел вида $p_{i}=2^{m}-1$. Так как все числа Мерсенна составляют подмножество множества $\left\{2^{m}-1\right\}_{m=1}^{\infty}$, то все выводы справедливы и для взаимно простых чисел Мерсена. Следовательно, согласно (7) и (8), необходимая длина двоичной кодограммы для представления числа из диапазона

$$
P=\prod_{i=1}^{L}\left(2^{n_{i}}-1\right)-1,
$$

где $n_{i}$ - простые числа, составит

$$
k(L)=\left\lceil\log _{2} \prod_{i=1}^{L}\left(2^{n_{i}}-1\right)\right\rceil=\sum_{i=1}^{L} n_{i},
$$

т.е. будет определяться суммой длин кодограмм необходимых для представления остатков числа того же диапазона в СОК. На основании этого можно сформулировать следующую теорему.

Теорема 2. Скорость передачи информации в двоичных каналах связи остается неизменной как в позичионной системе счисления, так и в системе остаточных классов при использовании оснований $p_{i} \leqslant 2^{i}$.

Важным выводом теоремы 2 является то, что в двоичных $\mathrm{KC}$ при передаче данных формата СОK для предотвращения потери скорости передачи информации необходимо использовать только основания, полученные из выражения $p_{i}=2^{i}-x$, где $x \geqslant 0$. Численные расчеты скорости и передачи информации можно осуществлять у учетом вероятности вычетов в COK (см. [7]).

Таким образом, последовательные КС можно использовать для передачи параллельных данных, представленных в формате СОК, без потери скорости передачи информации. Важным выводом теоремы 1 является тот факт, что передача информации в формате COK по двоичным КС всегда осуществляется с избыточностью. Однако, поскольку диапазон представления чисел в формате $\mathrm{COK}(1)$ не кратен $2^{m}$, то передача того же диапазона чисел в формате ПСС по двоичным КС будет осуществляться так же с информационной избыточностью, обусловленной процедурой округления. Оценим значение этих избыточностей.

Количество информации, содержащейся в кодограмме, определяется согласно выражению $H=\log _{2} M$ [4], где $M$ - объем алфавита кодограмм, максимальное количество комбинаций, которое возможно передать с использованием данного кода. С учетом нулевой кодограммы объем алфавита определяется через числовой диапазон как $M=P+1$. Поскольку в СОК утрачена информация о позиции каждого элемента, логично предположить, что информационная производительность ИС в этом формате должна превышать ту же производительность в формате ПСС. Оценим эту разницу.

Для определения информационной избыточности при кодировании данных COK в двоичной ПСС предположим, что основания могут быть любыми, в том числе и кратными $2^{m}$. Тогда максимальный вычет, кодируемый $n_{i}$ битами, составляет $\alpha_{i}=2^{n_{i}}-1$, т.е. основания могут быть равными $p_{i}=2^{n_{i}}$, а максимальный числовой диапазон представимый этими основаниями будет равен

$$
P^{\prime}=\prod_{i=1}^{L} 2^{n_{i}}-1 .
$$

Однако реально наиболее близкими основаниями к числам $2^{m}$, как уже отмечалось, являются числа $p_{i}=2^{n_{i}}-1$. Поэтому максимальный числовой диапазон по таким основаниям составит (10), 
что меньше $P^{\prime}$. Количество информации, содержащейся в данной избыточности, определяется как

$$
H_{s}=\log _{2}\left(P^{\prime}+1\right)-\log _{2}(P+1)=\sum_{i=1}^{L} \log _{2} 2^{n_{i}}-\sum_{i=1}^{L} \log _{2}\left(2^{n_{i}}-1\right)=\log _{2}\left(\prod_{i=1}^{L} \frac{2^{n_{i}}}{2^{n_{i}}-1}\right) .
$$

Численные расчеты показывают, что даже для натуральных $n_{i}=2,3,4, \ldots$ и $L \rightarrow \infty$ количество такой информации не составит больше $H \leqslant 0,5 \log _{2} 3<2$ бит. Для определения информационной избыточности двоичной ПСС при кодировании чисел диапазона (10) заметим, что необходимая длина кодограммы, согласно (11), составляет

$$
n=\sum_{i=1}^{L} n_{i}
$$

Максимальное число, представимое этой длиной, составит

$$
P^{\prime \prime}=2^{n}-1=\prod_{i=1}^{L} 2^{n_{i}}-1
$$

в то время как используется только числовой диапазон (10). Очевидно, что $P^{\prime}=P^{\prime \prime}$, т.е. информационная избыточность при кодировании чисел диапазона $P$ как в системе $\mathrm{COK}$, так и в системе ПСС одинакова. На основании последних рассуждений можно сформулировать следующую теорему.

Теорема 3. Энтропия непозиционных систем счисления равна энтропии ПСС. Информация, содержащаяся в значениях позиций элементов, расходуется на возможность осуществления параллельных операчий.

Рассмотрим в качестве оснований числа Ферма. Как отмечалось, максимальные вычеты по числам Ферма $2^{2^{m}}+1$ могут быть закодированы с максимальной избыточностью, так как округление осуществляется в большую сторону, кратную $2^{m}$. Поэтому в отличие от (6) получим

$$
\begin{aligned}
n(L)=\sum_{i=0}^{K}\left\lceil\log _{2} p_{i}\right\rceil=\sum_{m=0}^{K}\left\lceil\log _{2}\left(2^{2^{m}}+1\right)\right\rceil= \\
\quad=\sum_{m=0}^{K} \log _{2} 2^{2^{m}+1}=\sum_{m=0}^{K}\left(2^{m}+1\right)=\sum_{m=0}^{K} 2^{m}+K+1=2^{L}+L
\end{aligned}
$$

где $K=L-1=0,1,2, \ldots$. Таким образом, каждый максимальный вычет по модулю Ферма кодируется на один бит больше, чем $2^{m}$, образуя дополнительную информационную избыточность. Например, для передачи всех вычетов по основанию $2^{2^{2}}+1=17$, т.е. всех чисел от 0 до 16 , необходимо $n=\log _{2}(16+1)=5$ элементарных посылок. Однако максимальное число цифр, включая нуль, передаваемых кодом длинной $k=5$, составляет $P=2^{k}-1=31$. Таким образом, в отличие от чисел Мерсена, кодирование вычетов по основаниям Ферма осуществляется с максимальной избыточностью. Заметим, что числа Ферма обладают следующим свойством:

$$
\prod_{m=0}^{K}\left(2^{2^{m}}+1\right)=2^{2^{K+1}}-1=2^{2^{L}}-1=2^{T}-1,
$$

где $T=2^{L}$, т.е. последующее число Ферма определяется произведением предыдущих чисел. Так, для первых трех чисел Ферма $3 \cdot 5 \cdot 17=255$, а следующее число Ферма $257=255+2=256+1=$ $2^{2^{3}}+1$, т.е. числовой диапазон по основаниям чисел Ферма с учетом (13)

$$
P_{F}=\prod_{m=0}^{K}\left(2^{2^{m}}+1\right)=2^{2^{K+1}}-1
$$


может быть закодирован двоичным кодом длиной

$$
k(L)=\log _{2}(P+1)=\log _{2}\left[\prod_{m=0}^{K}\left(2^{2^{m}}+1\right)+1\right]=\log _{2} 2^{2^{L}}=2^{L},
$$

или без информационной избыточности. В то же время для кодирования всех максимальных вычетов, определяемых по основаниям чисел Ферма $\left(\alpha_{i}=p_{i}-1=2^{2^{m}}\right)$, согласно $(12)$, необходимо

$$
n(L)=\sum_{m=0}^{K} 2^{m}+K+1=2^{L}+L
$$

бит кода. Очевидно, что при $L \geqslant 1$ справедливо $n(L)>k(L)$, т.е. код в формате COK по основаниям чисел Ферма, с учетом (14), будет длиннее на $n(L)-k(L)=L+1=K$ бит, чем тот же код в формате ПСС, что соответствует снижению скорости передачи данных COK по отношению тех же данных в формате ПСС.

Последнее заключение является доказательством условия теоремы 2 , которая не справедлива для чисел Ферма вследствие того, что эти числа не удовлетворяют условию $p_{i} \leqslant 2^{i}$.

Избыточность в этом случае кода в ПСС $H_{p}=0$, в то время как избыточность кода в $\mathrm{COK}$ составит

$$
H_{s}=\log _{2}\left(P_{F}+1\right)-\log _{2} 2^{n(L)}=\log _{2} 2^{2^{L}}-\log _{2}\left(2^{L}+L\right)=\log _{2}\left(\frac{2^{2^{L}}}{2^{L}+L}\right) .
$$

Числовые расчеты, согласно (15), показывают, что информационная избыточность СОК по основаниям чисел Ферма в двоичном $\mathrm{KC}$ неограниченно возрастает по мере увеличения количества $L$ этих оснований. Так, при использовании трех $(L=3)$ оснований $p_{i}=3,5,7$ информационная избыточность составит $H_{s} \approx 4,5$ бит, причем эта избыточность обусловлена округлением каждого двоичного кода основания до целого числа и не превышает единицы на каждом из них. Поэтому для использования этой избыточности необходимы дополнительные методы кодирования.

3. Выводы. В результате приведенных рассуждений можно сделать следующие выводы.

1. Максимальный вычет любого простого числа кодируется в двоичном коде как минимум с избыточностью в одну кодограмму.

2. Скорость передачи информации в двоичных каналах связи остается неизменной как в позиционной системе счисления, так и в системе остаточных классов при использовании оснований $p_{i} \leqslant 2^{i}$.

3. Энтропия непозиционных систем счисления равна энтропии ПСС.

4. Информация, содержащаяся в значениях позиций элементов, расходуется на возможность осуществления операций без переноса.

5. Применение в качестве оснований чисел Ферма приводит к снижению скорости передачи информации по двоичным КС.

\section{СПИСОК ЛИТЕРАТУРЫ}

1. Акушский И. Я., Пак И. Т. Вопросы помехоустойчивого кодирования в непозиционном коде// Вопр. киберн. - 1977. - 28. - С. 36-56.

2. Виноградов И. М. Основы теории чисел. - М.: Наука, 1972.

3. Макклеллан Дж. Г., Рейдер Ч. М. Применение теории чисел в цифровой обработке сигналов. - М.: Радио и связь, 1983.

4. Скляр Б. Цифровая связь. Теоретические основы и практическое применение. - М.: Вильямс, 2003.

5. Смирнов А. А., Плетнев И. Н., Уруджев У. Р. Помехоустойчивость параллельных каналов связи в системе остаточных классов// в кн.: Проектирование и технологии электронных средств. - ВЛГУ, 2016. - C. $45-48$.

6. Смирнов А. А., Бондарь В. В., Белоконь Л. В., Саиег Т. Х., Даржания А. Д., Роженко О. Д., Мирзоян М. В., Смирнова О. Н. Преобразование Фурье и Уолша дискретных сигналов в системе остаточных классов// Междунар. ж. прикл. фундам. исслед. - 2018. - № 1. - С. 36-40. 
7. Смирнов А. А., Саиег Т. Х., Даржания А. Д., Роженко О. Д., Смирнова О. Н. Распределение вероятностей вычетов в системе остаточных классов// Соврем. наукоемк. технол. - 2017. - № 7. - С. 78-82.

8. Червяков Н. И., Сахнюк П. А., Шапошников А. В., Ряднов С. А. Модулярные параллельные вычислительные структуры нейропроцессорных систем. - М.: Физматлит, 2003.

9. Червяков Н. И., Коляда А. А., Ляхов П. А., Бабенко М. Г., Лавриненко И. Н., Лавриненко А. В. Модулярная арифметика и ее приложения в инфокоммуникационных технологиях. - М.: Физматлит, 2017.

Смирнов Александр Александрович

Северо-Кавказский федеральный университет, Ставрополь

E-mail: shursun@mail.ru

Бондарь Виктория Витальевна

Северо-Кавказский федеральный университет, Ставрополь

E-mail: viktori-bondar@yandex.ru

Роженко Ольга Дмитриевна

Северо-Кавказский федеральный университет, Ставрополь

E-mail: r.o.d@mail.ru

Мирзоян Марине Вагановна

Северо-Кавказский федеральный университет, Ставрополь

E-mail: vaganovna73@mail.ru

Даржания Анна Дмитриевна

Северо-Кавказский федеральный университет, Ставрополь

E-mail: anna22051@yandex.ru 